in photo-electric recording of spectra from transient sparks. The techniques have been developed specifically to improve time-resolved spectroscopic analysis of solids, for example, the quantitative detection of copper in aluminium alloys. The controlled gating of the photo-multiplier proved to be so good that special controlled spark sources had to be developed and a description of such a source was given based on the work of A. Bardocz in Hungary. This source used an air-blasted control gap in series with the test gap. The jitter proved to be not more than 15 musec. over $10^{7}$ discharges, and the importance of ultra-violet illumination of the test gap by the control gap was shown to be important.

It was possible to analyse a spectral line with a resolving time of $0.1 \mu$ sec. The speaker stressed that in spectroscopic analysis of this kind time-resolved observations might well be of importance in the investigation of analysis and internal standard lines, etc.

Dr. C. Breton (Fontenay-aux-Roses) gave an account of spectroscopic observations made in that establishment, including the observation of impurity lines in the far ultre-violet in a toroidal discharge and a new technique for the study of line profiles. The former work was carried out with a vacuum grating spectrograph using seven exit slits, each with a separate photomultiplier tube. Impurity lines from carbon, oxygen and nitrogen ions were observed with a $20 \mathrm{kV}$. 200 microfarad capacity bank feeding toroidal discharges at a pressure of $10^{-\mathbf{a}} \mathrm{mm}$. of mercury. Dr. Breton deseribed a new and interesting technique in which a broadened line profile is divided into thin slices by the use of successive layers of glass fibres some $50 \mu$ in diameter to centres of spaces approximately $55 \mu$ apart. The assembly is used in a 1-m. focal distance monochromator giving an average dispersion of about $3 \AA$. $/ \mathrm{mm}$. in the first order and in the visible region. Each fibre corresponds to an interval of about $0.15 \AA$. and Dr. Breton showed curves giving temporal variations of the profile of $H_{\beta}$; finally, he mentioned experiments on plasma radiation and the drift velocity of ions in a magnetic mirror machine.
J. D. Craggs

\title{
ELECTRON SPIN RESONANCE
}

$\mathrm{O}^{\mathrm{N}}$ $\mathrm{N}$ November 2 the Chemical Society held a symposium at Queen Mary College, London, on electron spin resonance. In the first part of the symposium there were three papers. In an introductory paper, Prof. H. C. Longuet-Higgins outlined the principles of electron spin resonance measurements. Only paramagnetic substances show this phenomenon, which arises from their unpaired electrons. In a magnetic field the two spin states of a single electron are split apart in energy by virtue of the magnetic moment of the electron. An oscillating magnetic field, applied at right angles to the permanent field, causes the electron to jump from one of these levels to the other if the frequency $v$ of the oscillating field satisfies the relationship:

$$
h_{v}=g \beta H, \beta=e h / 4 \pi m c
$$

Here $H$ is the strength of the permanent field and $g$ is a number which equals 2.0023 for a free electron. Electron spin resonance measurements acquire their interest from two subsidiary physical effects. The first of these is the so-called hyperfine interaction, which arises when the paramagnetic molecule includes magnetic nuclei. The value of $g$ then depends on the orientation of all the magnetic nuclei, and a series of lines is observed rather than a single resonance. As an example, $n$ equivalent protons will give rise to $n+1$ hyperfine lines with a binomial distribution of intensity; a single nucleus of spin $I$ (which may be $\frac{1}{2}, 1, \frac{3}{2}$, etc.) will give a set of $2 I+1$ equally spaced lines of equal intensity. The foregoing remarks apply to a paramagnetic molecule tumbling over and over in the liquid phase. But if the molecule is fixed in the external magnetic field, the spectrum may be more complicated because of direction-dependent interactions between the electron and the magnetic nuclei. The directional variation of the spectrum may then enable one to identify the orbital in which the odd electron is moving.

The other physical effect which provides useful information about the nature of the unpaired electrons is spin-orbit coupling. An electron with orbital angular momentum is easier to orientate in some directions than in others, and this means that $g$ must be regarded not as a pure number but as a tensor. The axes of the $g$ tensor must coincide with the symmetry axes of the molecule, and certain applications of this fact would be discussed later in the sympo. sium.

The next paper, by Prof. S. I. Weissman, was concerned with line-widths in electron resonance spectra. The width of a line is inversely proportion al to the mean life-time of the spin states involved in the transition. Measurements of line-broadening can therefore throw light on the rate at which electrons are transferred from one molecule to another (assuming this to be the process which determines the lifetime). Prof. Weissman gave some examples, including the reaction of the naphthalene molecule with its negative molecule ion and the exchange of a sodium ion between two naphthalene molecule ions. As he pointed out, an electron resonance spectrum always supplies kinetic information, whether one is interested in it or not.

The third paper, by Prof. D. J. E. Ingram, was concerned with the structural information which is provided by electron resonance measurements on single crystals. Prof. Ingram classified this information under three headings: measurements on the $g$ tensor, studies of the hyperfine structure and measure. ments of electronic splittings (sometimes called zerofield splittings). At Southampton he and his colleagues had determined the orientation of the hæm in groups in myoglobin and hæmoglobin by examining the way in which $g$ varies when the crystal is rotated in the externally applied magnetic field. In this particular case there is a very large variation in $g$, from 2 to 6 , so that the orientation of the hæms can be very accurately determined. In myoglobin there are two hæms per unit cell and their orientetion as determined in this way was afterwards confirmed by the X-ray work of $\mathbf{J}$. C. Kendrew. An interesting molecule is hæmoglobin azide, where the $g$ 
tensor is completely anisotropic. J. S. Griffith had been able to determine from this tensor the order and spacing of the $3 d$ orbitals on the iron atom; this order indicates very strongly that the histidine group attached to the iron atom is in a plane parallel to one of the diagonals of the hæm plane.

Information can also be obtained from the hyperfine splittings produced by protons or other magnetic nuclei. These splittings can be correlated with the types of group that are present and the angles which they make with one another. In order to extract such information from the data, one must, however, make use of molecular orbital theory, and the same applies to the sort of information which can be obtained from measurements of zero-field splittings.

In the second section of the symposium, four papers were presented.

The first paper, by J. R. Bolton and A. Carrington, was concerned with two topics. The first was the measurement and interpretation of the electron spin resonance spectra of the negative ions of toluene, $p$-xylene and $m$-xylene. These are of special interest because of the degeneracy of the lowest antibonding orbitals of benzene. This degeneracy is removed by introducing methyl groups, and one can predict from the molecular orbital theory which of the two orbitals is likely to be occupied in the ground-state of each ion. The spectra of these xylenes, and of their partially deuterated isotopes, completely confirm the theoretical predictions, and in particular the hypothesis that the mothyl groups repel the additional electron.

The other topic discussed by Dr. Carrington was certain remarkable line-width alternation effects which occur in the electron spin resonance spectra of the durosemiquinone positive ion and in the positive ion of 5,8-dihydroxy-1,4-naphthasemiquinone. This effect is almost certainly due to cis-trans isomerization proceeding at a rate comparable with the separation (in cycles/sec.) between the hyperfine lines in the resonance spectra. Dr. Carrington also directed attention to the possibility of determining the signs of hyperfine interaction constants by studying the variation of line width as one proceeds from one end of a spectrum to the other.

In the next paper Dr. E. A. C. Lucken described experiments on various substituted $p$-benzosemiquinones and polynuclear aromatic quinones. He used these measurements in attempting to evaluate coulomb integrals and resonance integrals for the various types of atoms and bonds. These attempts were not altogether successful, but Dr. Lucken claimed that his experiments could be inter. preted by supposing that the methyl group produced an inductive effect, but did not enter into hyperconjugation. In the subsequent discussion, Dr. Carrington said that he, J. R. Bolton and A. D. McLachlan had obtained strong evidence for hyperconjugationat least in hydrocarbon cations-from experiments on the positive and negative ions of anthracene and its 9-methyl and 9,10-dimethyl derivatives.

Prof. M. C. R. Symons then gave a lecture on the use of electron resonance in identifying the products of radiation damage in some inorganic oxy-salts. As Prof. Symons pointed out, such work can provide interesting information about the closed-shell species from which the paramagnetic radicals or radical ions are derived. He illustrated this remark with reference to the manganate and hypomanganate ions which result from the addition of one or two electrons to $\mathrm{MnO}_{4}{ }^{-}$. Work on damaged crystals is, however, often complicated by the difficulty of identifying the products, especially if the common isotopes of the nuclei have no magnetic moment. Nevertheless, in some cases it may be possible to argue by analogy, as in the series $\mathrm{ClO}_{3} . \mathrm{SO}_{3}^{-}, \mathrm{PO}_{3}^{--}$. Prof. Symons described some preliminary work on the oxyions of chlorine, nitrogen and sulphur, and offered an explanation for some apparent discrepancies between the results of various authors who had studied the $\mathrm{NO}_{2}$ molecule in different environments.

The final paper in the symposium was given by J. R. Morton. When a crystal of L-alanine is irradiated with $\gamma$-rays the radical $\mathrm{CH}_{3} \mathrm{CHCO}_{2} \mathrm{H}$ is produced. The electron spin resonance spectrum of this radical shows hyperfine structure which varies with the orientation of the crystal and also changes markedly when the crystal is cooled to $77^{\circ} \mathrm{K}$. It is possible to analyse this spectrum fully and to obtain the hyperfine coupling tensors for the $\mathrm{CH}$ proton and the three protons of the $\mathrm{CH}_{8}$ group. The radical is found to be planar, with a $\mathrm{CH}_{3}-\mathrm{C}-\mathrm{H}$ angle of $121 \pm 3^{\circ}$. The spectra at $77^{\circ} \mathrm{K}$. indicate that the methyl group has ceased to rotate at that temperature, and an estimate of the height of the rotation barrier can be obtained from the spectra at intermediate temperatures.

At the end of the meeting Dr. D. H. Whiffen proposed a vote of thanks to Prof. K. W. Sykes for arranging the symposium and to Queen Mary College, London. for generous hospitality.

\section{THE HEALTH OF SEAFARERS}

\begin{abstract}
$\mathrm{T}$ THE total number of seafarers on ships other than those engaged in coastal trading is in the region of 750,000. They form a definite community the members of which are linked together by mutual interest-and health hazards-at least as closely linked as they are to their parent countries. Although much has already been done to improve their working conditions and health standards, much remains to be done. In 1959 the World Health Organization undertook a study of the whole problem of the health of seafarers. As a preliminary fact-finding step, the World Health Organization Regional Office for Europe convened a conference at which it became
\end{abstract}

clear that not enough was known of the health problems of seafarers or of the health services available to them. Following this conference, a questionnaire was drewn up by the Organization in consultation with the International Labour Organization and circulated to member States. The next step was to appoint a consultant to visit some of the major ports to analyse the problem in those ports where services were already in being and to supplement, where necessary, the information given in replies to the questionnaire. The ports visited were Liverpool, London, Marseilles, Athens, Alexandria, Bombay, Singapore, Manila, Hong Kong, Tokyo, San Fran- 